Journal of Organometallic Chemistry, 431 (1992) 151-157

Elsevier Sequoia S.A., Lausanne

JOM 22555

\title{
Untersuchungen zum Einfluß des Biscyclopentadienylmethanliganden auf die Bildung von Cyclopentadienylchromsulfiden
}

\author{
Henri Brunner, Roland Graßl und Joachim Wachter \\ Institut für Anorganische Chemie der Universität Regensburg, Universitätsstraße 31, W-8400 Regensburg \\ (Deutschland)
}

(Eingegangen den 29. November 1991)

\begin{abstract}
The reaction of bcpmCr $\mathrm{r}_{2}(\mathrm{CO})_{6}(\mathrm{bcpm}=$ biscyclopentadienylmethane) with sulfur gives in a stepwise reaction the complexes bcpmCr $2(\mathrm{CO})_{4} \mathrm{~S}(2)$ and bcpm ${ }_{2} \mathrm{Cr}_{4} \mathrm{~S}_{4}(3)$. The products were investigated by IR and low temperature ${ }^{1} \mathrm{H}$ NMR spectroscopy. The ${ }^{1} \mathrm{H}$ NMR spectra of 2 show upon cooling a dynamic process similar to that in the starting material. It is ascribed to a topomerization process in the bridging ligand. The cluster 3 which belongs to the heterocubane type exhibits a magnetic behavior which is inbetween that of the antiferromagnetic clusters of type $\mathrm{Cp}_{4} \mathrm{Cr}_{4} \mathrm{O}_{4}\left(\mathrm{Cp}=\right.$ any substituted $\eta^{5}-\mathrm{C}_{5} \mathrm{H}_{5}$ ring) and the diamagnetic $\mathrm{Cp}_{4} \mathrm{Cr}_{4} \mathrm{~S}_{4}$ cubanes.
\end{abstract}

\section{Zusammenfassung}

Die Reaktion von bcpmCr $_{2}(\mathrm{CO})_{6}$ (bcpm $=$ Biscyclopentadienylmethan) mit Schwefel verläuft stufenweise unter Bildung der Komplexe bcpmCr $2(\mathrm{CO})_{4} \mathrm{~S}$ (2) und $\mathrm{bcpm}_{2} \mathrm{Cr}_{4} \mathrm{~S}_{4}$ (3). Die Produkte wurden durch IR- und Tieftemperatur- ${ }^{1} \mathrm{H}$-NMR-Spektroskopie untersucht. Die ${ }^{1} \mathrm{H}$-NMR-Spektren von 2 zeigen beim Abkühlen ein ähnliches dynamisches Verhalten wie das Edukt. Dies wird einem Topomerisierungsproze $B$ im Brückenliganden zugeschrieben. Der dem Heterocubantyp zuzuordnende Cluster 3 weist ein magnetisches Verhalten auf, das zwischen den von antiferromagnetischen Clustern vom Typ $\mathrm{Cp}_{4} \mathrm{Cr}_{4} \mathrm{O}_{4}\left(\mathrm{Cp}=\right.$ beliebig substituierter $\eta^{5}-\mathrm{C}_{5} \mathrm{H}_{5}$-Ring) und den diamagnetischen $\mathrm{Cp}_{4} \mathrm{Cr}_{4} \mathrm{~S}_{4}$ Cubanen liegt.

\section{Einleitung}

Die Reaktion von elementarem Schwefel mit reaktiven Cyclopentadienylchrom-Komplexfragmenten führt zur Bildung einer ganzen Reihe von strukturell interessanten $\mathrm{CpCr}$-Sulfiden mit hohem Schwefelgehalt [1]. Reaktionsablauf und Natur der Endprodukte werden stark von den Eigenschaften der Startkomplexe bestimmt. Einmal läßt sich über die formale Substitution aller CO-Liganden aus

Correspondence to: Dr. J. Wachter, Institut für Anorganische Chemie der Universität Regensburg, Universitätsstraße 31, W-8400 Regensburg, Deutschland. 

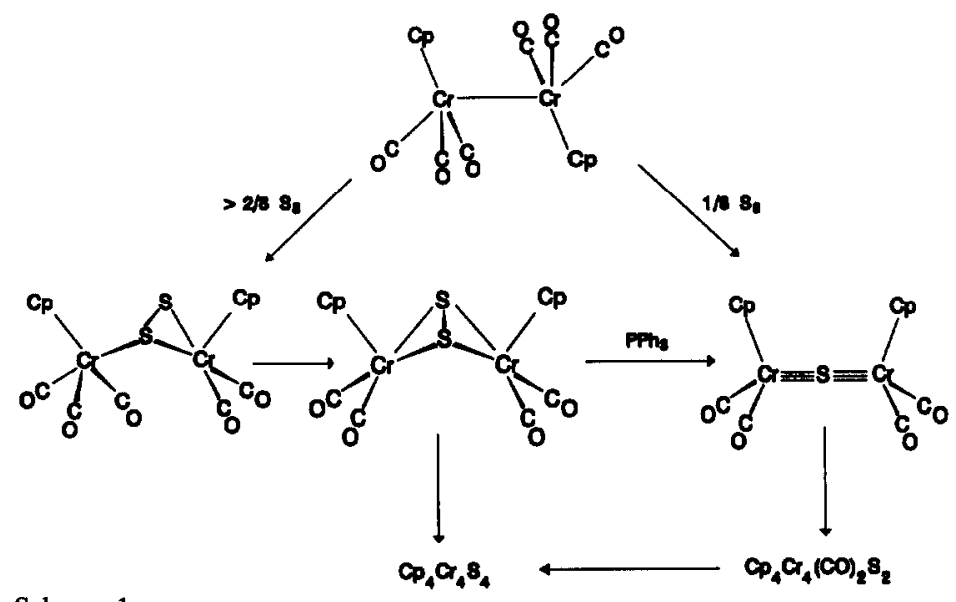

Schema 1.

$\mathrm{Cp}_{2}^{\star}{ }_{2} \mathrm{Cr}_{2}(\mathrm{CO})_{4}(\mathrm{Cr} \equiv \mathrm{Cr})\left(\mathrm{Cp}^{\star}=\eta^{5}-\mathrm{C}_{5} \mathrm{Me}_{5}\right)$ durch Schwefel der Komplex $\mathrm{Cp}_{2}^{\star}{ }_{2} \mathrm{Cr}_{2} \mathrm{~S}_{5}$ darstellen [2]. Diese Reaktion verläuft wohl über CO-haltige Zwischenstufen, die sich jedoch im Gegensatz zur analogen Mo-Chemie [1,3] hier nicht nachweisen lassen. Eine Abhängigkeit der Reaktion vom Cp-Substituenten wurde bisher nicht untersucht. Ein zweiter Reaktionstyp nutzt die leichte Spaltbarkeit der $\mathrm{Cr}-\mathrm{Cr}$-Bindung im Komplex $\left(\mathrm{C}_{5} \mathrm{H}_{5}\right)_{2} \mathrm{Cr}_{2}(\mathrm{CO})_{6}$, indem Schwefel stufenweise in die M-M-Bindung eingeschoben wird (Schema 1) [4]. Analoge Produkte werden in der Reaktion von $\mathrm{Cp}{ }^{\star} \mathrm{Cr}(\mathrm{CO})_{3} \mathrm{~K}$ mit Trithiazylchlorid, $\mathrm{S}_{3} \mathrm{~N}_{3} \mathrm{Cl}_{3}$, gebildet [5]. Nachfolgende CO-Substitution und Dimerisierung führen zu den vom Cubantyp abgeleiteten Clustern $\mathrm{Cp}_{4} \mathrm{Cr}_{4} \mathrm{~S}_{4}$ als thermisch stabilste Komplexe [6].

Wenig Augenmerk wurde bisher auf die Untersuchung des Einflusses weiterer substituierter $\mathrm{Cp}$-Liganden gelegt. Hier schien uns vor allem der Biscyclopentadienylmethan(bcpm)-Komplex bcpmC $\mathrm{r}_{2}(\mathrm{CO})_{6}(1)$ interessant, der seit kurzem als Edukt zur Verfügung steht [7]. Die durch die Verklammerung der beiden Metallzentren erzielten kooperativen Effekte [8] sollten durchaus Auswirkungen auf die Natur und Stabilität der resultierenden Produkte haben.

\section{Präparative und spektroskopische Ergebnisse}

bcpmCr $\mathrm{Cr}_{2}(\mathrm{CO})_{4} \mathrm{~S}(2)$

Bei der Umsetzung von bcpmCr ${ }_{2}(\mathrm{CO})_{6}(1)$ mit $1 / 8$ Moläquivalent $S_{8}$ in Toluol ändert sich die Farbe der anfangs grünen Reaktionslösung rasch nach braun. Nach 4 h Rühren bei Raumtemperatur läßt sich nach chromatographischer Reinigung das Schwefelinsertionsprodukt bcpmCr ${ }_{2}(\mathrm{CO})_{4} \mathrm{~S} \mathrm{(2)}$ in $86 \%$ Ausbeute isolieren. Die Zusammensetzung dieser Verbindung wurde durch den Molekülionenpeak $m / e=$ $390.2 \mathrm{im}$ FD-Massenspektrum gesichert. Jedoch konnten wegen der offensichtlich langsamen Zersetzung in Lösung selbst bei $-18^{\circ} \mathrm{C}$ von bcpmCr ${ }_{2}(\mathrm{CO})_{4} \mathrm{~S} \mathrm{(2)} \mathrm{keine}$ einheitlichen Elementaranalysen erhalten werden.

Die unverhältnismäßig hohe Zahl von sechs $\nu(\mathrm{CO})$-Streckschwingungen im Infrarotspektrum von 2, aufgenommen in $\mathrm{KBr}$, reduziert sich in Toluol auf drei 
Banden, andeutungsweise ist eine vierte Bande in Form einer Schulter zu erkennen. Damit ähnelt 2 in Bandenlage und -charakteristik der unverbrückten Vergleichsverbindung $\mathrm{Cp}_{2} \mathrm{Cr}_{2}(\mathrm{CO})_{4} \mathrm{~S}$ sehr stark, für die eine gestaffelte CO-Konformation gefunden wurde $[4,9]$.

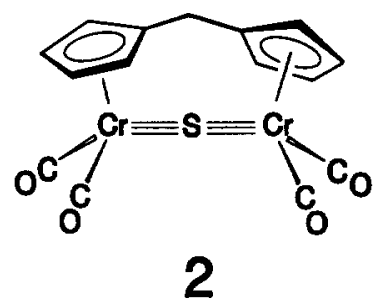

Dem ${ }^{1}$ H-NMR-Spektrum sind in Toluol- $d_{8}$-Lösung bei $297 \mathrm{~K}$ für die Methylenprotonen von Biscyclopentadienylmethan ein Singulett bei $2.03 \mathrm{ppm}$, für die Ringprotonen zwei pseudo-Tripletts bei 4.03 und $4.26 \mathrm{ppm}$ zu entnehmen. Beim Abkühlen erleidet vor allem das Signal bei $4.03 \mathrm{ppm}$ eine auffällige Veränderung: es verbreitert sich sichtlich bei $223 \mathrm{~K}$, um bei $193 \mathrm{~K}$ an deutlich hochfeldverschobener Stelle aufzutauchen. Das Signal bei $4.26 \mathrm{ppm}$ wird dagegen nur relativ wenig nach hohem Feld verschoben, auch verbreitert es sich nur unbedeutend (Fig. 1).

Damit liegt im Prinzip ein zum Eduktkomplex ähnliches Temperaturverhalten vor mit wohl relativ ähnlichen Energieparametern. Als einzige Abweichung kann im Tieftemperaturgrenzspektrum keine Aufspaltung in die für das Edukt typischen vier Protonensorten erreicht werden. Dies dürfte eine Folge der Aufweitung des $\mathrm{Cr}$-Cr-Abstandes von 3.281 [9] auf $c a .4 .0 \AA$ in 3 (vorausgesetzt nahezu linearer Aufbau mit hohem Mehrfachbindungsanteil wie in $\left.\left(\mathrm{C}_{5} \mathrm{H}_{5}\right)_{2} \mathrm{Cr}_{2}(\mathrm{CO})_{4} \mathrm{~S}\left[10^{*}\right]\right)$ sein, womit Wechselwirkungen zwischen den Ringprotonen und den CO-Liganden erschwert würden [8]. Somit ist die Topomerisierung des bcpm-Liganden der einzig mögliche dynamische Proze $\beta$ in 2 . Eine Rotation um das $\mathrm{Cr} \equiv \mathrm{S} \equiv \mathrm{Cr}$-Bindungssystem wie in $\left(\mathrm{C}_{5} \mathrm{H}_{5}\right)_{2} \mathrm{Cr}_{2}(\mathrm{CO})_{4} \mathrm{~S}$ [9] ist durch die Einführung des Brückenliganden auszuschließen.

$b c p m_{2} \mathrm{Cr}_{4} \mathrm{~S}_{4}(3)$

Erhöht man bei der Reaktion von bcpmCr ${ }_{2}(\mathrm{CO})_{6}$ (1) mit Schwefel den Schwefelanteil auf $2 / 8$ Moläquivalente, so scheint sich zunächst am Reaktionsverlauf nichts zu ändern. Ein Farbumschlag von grün nach braun (Bildung von 2) ist zu beobachten. Erhöht man jedoch die Reaktionstemperatur auf $55^{\circ} \mathrm{C}$, so erhält man nach $18 \mathrm{~h}$ wieder eine grüne Lösung. Bei der Chromatographie des Reaktionsansatzes wird zunächst ein labiles, carbonylhaltiges Nebenprodukt isoliert. Dieses kann zwar nicht näher charakterisiert werden, deutet aber auf einen komplizierten Verlauf der Reaktion eventuell im Sinn von Schema 1 hin. Dem grünen, in gängigen Lösungsmitteln nur mäßig löslichen Hauptprodukt kommt laut FD-MS die Zusammensetzung bcpm ${ }_{2} \mathrm{Cr}_{4} \mathrm{~S}_{4}$ (3) zu. Es kann auch ausgehend von bcpmCr ${ }_{2}(\mathrm{CO})_{4} \mathrm{~S}(2)$ durch Reaktion mit $1 / 8 \mathrm{~S}_{8}$ in THF bei $50^{\circ} \mathrm{C}$

* Die Literaturnummer mit einem Sternchen deutet eine Bemerkung in der Literaturliste an. 


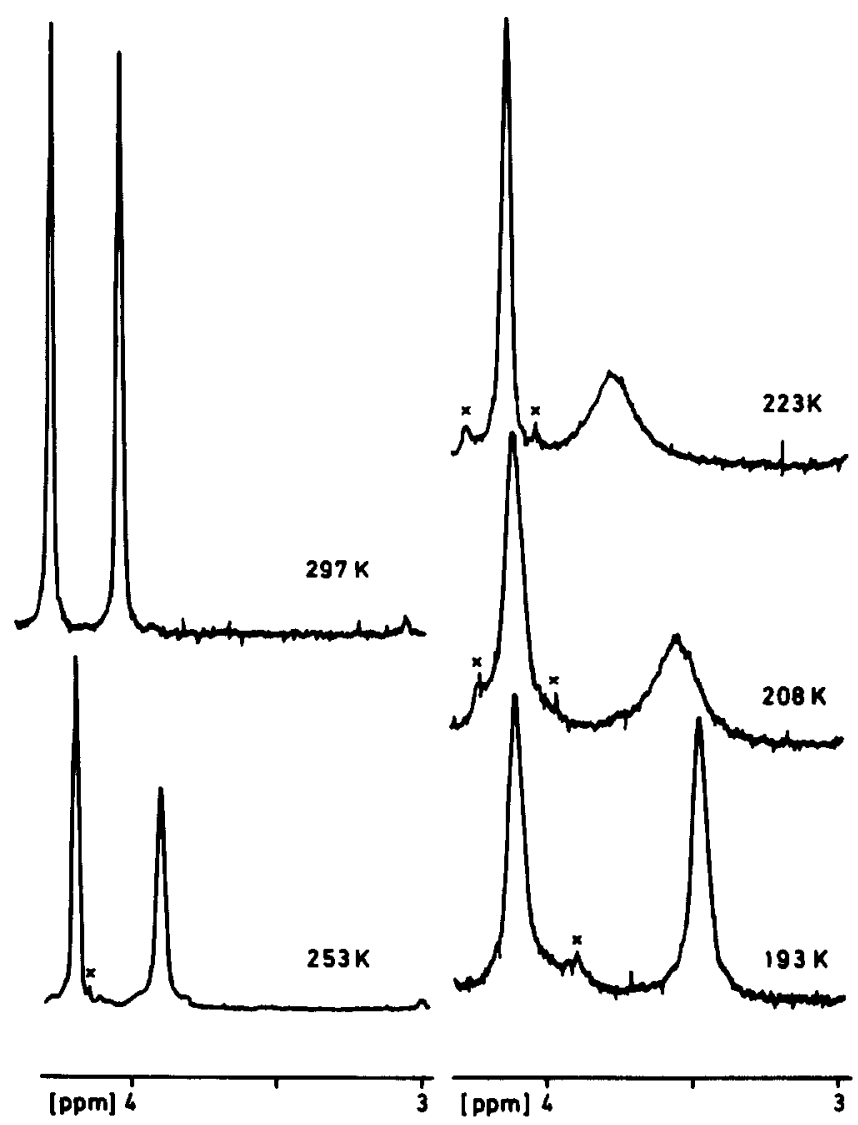

Fig. 1. Temperaturabhängige ${ }^{1} \mathrm{H}-\mathrm{NMR}-$ Spektren von 2 in Toluol- $d_{8}$ (i-TMS, $\left.250 \mathrm{MHz}\right)(\times$ markieren Verunreinigungen).

dargestellt werden. Die Ausbeute an 3 liegt mit $38 \%$ bei dieser Variante geringfügig höher.

Das IR-Spektrum von 3 enthält lediglich schwache Absorptionsbanden bei 462 und $421 \mathrm{~cm}^{-1}$, die $\mathrm{Cr}-\mathrm{S}$-Gerüstschwingungen zugeordnet werden, und die wenig informativen $\mathrm{C}-\mathrm{H}$ - bzw. C-C-Valenz- und Deformationsschwingungen des organischen Liganden in den Bereichen 3080, 2900, 1400 und $800 \mathrm{~cm}^{-1}$.

Interessant ist das ${ }^{1} \mathrm{H}$-NMR-Spektrum von 3 (Fig. 2): die Lage der Resonanzsignale der Ringprotonen im Raumtemperatur-Spektrum bei relativ tiefem Feld und ihre beträchtliche Temperaturabhängigkeit deuten auf einen gewissen antiferromagnetischen Beitrag in dieser Verbindung. Wesentlich weniger stark ausgeprägt ist der Temperaturshift des Singuletts der Methylenprotonen. Hinweise auf einen wie in 2 ablaufenden dynamischen Prozeß enthalten die Spektren nicht.

Strukturell ist 3 in die Klasse der Heterocubancluster $\mathrm{Cp}_{4} \mathrm{M}_{4} \mathrm{E}_{4}(\mathrm{E}=\mathrm{O}, \mathrm{S}, \mathrm{Se})$ einzureihen, die für $\mathrm{M}=\mathrm{Cr}$, Mo, W 60 Valenzelektronen und damit sechs MetallMetall-Bindungen aufweisen [11]. Diese Beschreibung ist für die Cr-Verbindungen durch Röntgenstrukturuntersuchungen an $\left(\mathrm{C}_{5} \mathrm{H}_{5}\right)_{4} \mathrm{Cr}_{4} \mathrm{~S}_{4}[12]$ und $\left(\mathrm{MeC}_{5} \mathrm{H}_{4}\right)_{4} \mathrm{Cr}_{4} \mathrm{~S}_{4}$ [13] bestätigt worden. Allen bisher beschriebenen Clustern mit S- bzw. Se-Ligan- 


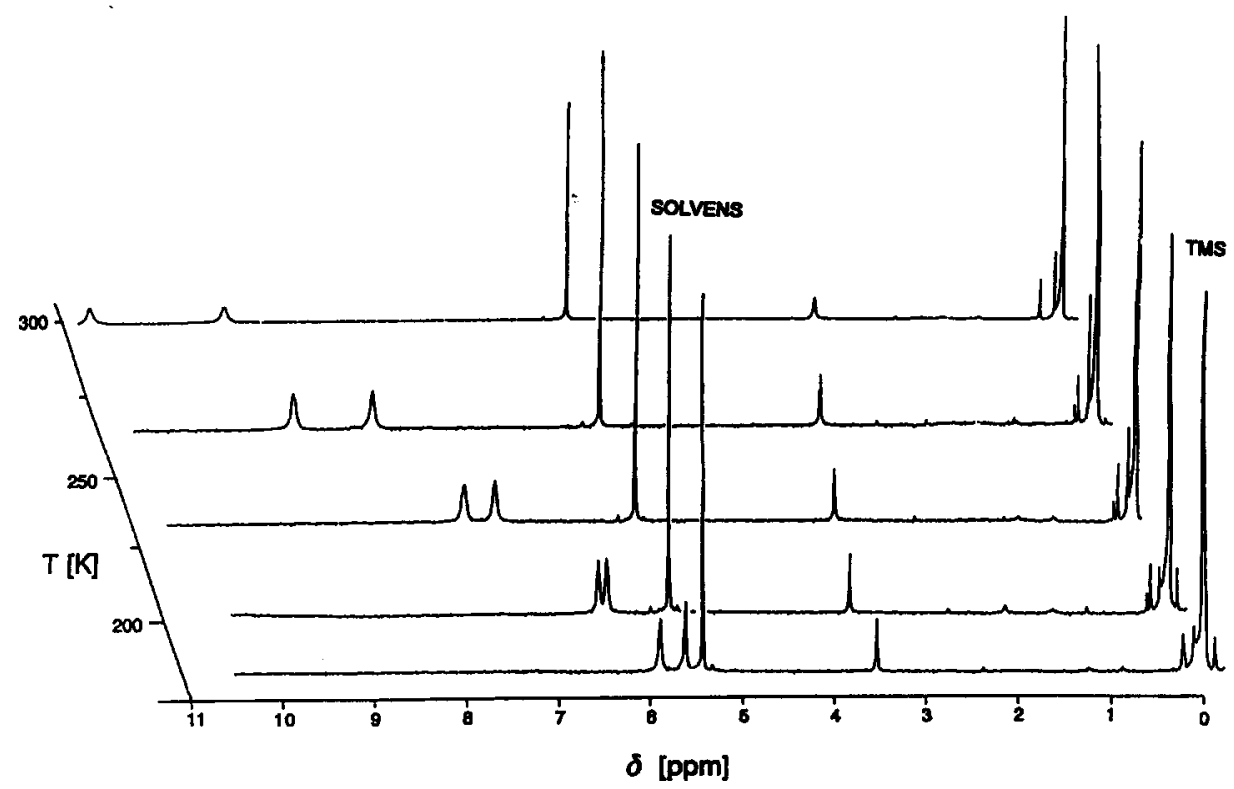

Fig. 2. Temperaturabhängige ${ }^{1} \mathrm{H}$-NMR-Spektren von 3 in $\mathrm{CD}_{2} \mathrm{Cl}_{2}$ (i-TMS, $250 \mathrm{MHz}$ ).

den ist Diamagnetismus gemeinsam [14], während Oxocubane des Typs $\mathrm{Cp}_{4} \mathrm{Cr}_{4} \mathrm{O}_{4}$ antiferromagnetisches Verhalten zeigen $[14,15]$. In letzteren üben Symmetriestörungen im Metalltetraeder einen bedeutenden Einfluß auf die energetische Lage der Clusterorbitale, die nur teilweise besetzt sind, aus. Wegen der Ähnlichkeit ihrer Energieniveaus ist die Besetzung von Orbitalen durch ungepaarte Elektronen thermisch möglich.

Das magnetische Moment in 3 nimmt von 1.58 auf $0.93 \mathrm{BM}$ im Temperaturbereich von $293-81 \mathrm{~K}$ ab [16* ${ }^{*}$; es ist somit bei weitem nicht so stark ausgeprägt wie in den $\mathrm{Cp}_{4} \mathrm{Cr}_{4} \mathrm{O}_{4}$-Clustern [14,15]. Dennoch liefert es einen signifikanten Hinweis auf eine Verzerrung des Clustergerüstes, die diesmal ihre Ursache in dem organischen Brückenliganden haben dürfte.

\section{Experimenteller Teil}

Sämtliche Arbeiten wurden unter Schutzgas $\left(\mathrm{N}_{2}\right)$ und unter Verwendung von trockenen, $\mathrm{N}_{2}$-gesättigten Lösungsmitteln durchgeführt. Als Meßgerät diente für die Aufnahme der ${ }^{1}$ H-NMR-Spektren ein Bruker WM 250-Spektrometer, für die der Infrarotspektren ein Beckman Infrarot-Gitterspektrometer Modell 4240. Verbindung 1 wurde nach bereits beschriebenem Verfahren erhalten [7].

Darstellung von bcpmCr ${ }_{2}(\mathrm{CO})_{4} S$ (2)

Die intensiv grün gefärbte Lösung von $690 \mathrm{mg}(1.67 \mathrm{mmol}) 1$ in ca. $80 \mathrm{ml}$ Toluol wird mit $54 \mathrm{mg}(1.67 \mathrm{mmol})$ Schwefel $4 \mathrm{~h}$ bei Raumtemperatur gerührt. Die nunmehr braune Reaktionslösung wird auf ca. $15 \mathrm{ml}$ eingeengt und an $\mathrm{SiO}_{2}$ mit Toluol (Säule $3 \times 25 \mathrm{~cm}$ ) chromatographiert. Eine braune Zone enthält 2 in $86 \%$ Ausbeute. Komplex 2 läßt sich aus Toluol umkristallisieren, liefert jedoch keine reproduzierbare Elementaranalysen. 
IR $\left(\mathrm{cm}^{-1}\right)$ 2: $\nu(\mathrm{CO})(\mathrm{KBr}): 2000 \mathrm{vs}, 1974 \mathrm{vs}, 1943 \mathrm{vs}, 1917 \mathrm{~s}, 1875 \mathrm{vs}, 1858 \mathrm{vs} ; \nu(\mathrm{CO})$ (Toluol): 2002vs, 1960vs, 1932vs, $1890 \mathrm{sh} ; \mathrm{C}_{15} \mathrm{H}_{10} \mathrm{Cr}_{2} \mathrm{O}_{4} \mathrm{~S}$ (390.29), Molmasse 390.20 (FD-MS aus Toluol).

Darstellung von bcpm ${ }_{2} \mathrm{Cr}_{4} \mathrm{~S}_{4}(3)$

(a) Umsetzung von bcpmCr ${ }_{2}(\mathrm{CO})_{6}$ (1) mit Schwefel. $420 \mathrm{mg}(1.01 \mathrm{mmol}) 1$ und $65 \mathrm{mg}(2.02 \mathrm{mmol})$ Schwefel werden in $80 \mathrm{ml}$ Toluol gelöst. Nach $18 \mathrm{~h}$ Rühren bei $55^{\circ} \mathrm{C}$ engt man die Reaktionslösung auf die Hälfte ihres Volumens ein und chromatographiert an $\mathrm{SiO}_{2}$ mit Toluol (Säule $3 \times 25 \mathrm{~cm}$ ). Eine erste, braungrüne Zone enthält eine nicht näher charakterisierbare, carbonylhaltige Spezies, die darauf folgende grüne Zone liefert 3 in 33\% Ausbeute.

(b) Umsetzung von bcpmCr ${ }_{2}(\mathrm{CO})_{4} S$ (2) mit Schwefel. Die braune Lösung von $530 \mathrm{mg}(1.36 \mathrm{mmol}) 2 \mathrm{in} 80 \mathrm{ml}$ THF wird mit $44 \mathrm{mg}(1.37 \mathrm{mmol})$ Schwefel versetzt und $6 \mathrm{~h}$ bei $50^{\circ} \mathrm{C}$ gerührt. Der nach Entfernen des Lösungsmittels verbleibende grüne Rückstand wird mit $50 \mathrm{ml}$ Toluol extrahiert. Die auf $c a .20 \mathrm{ml}$ eingeengte Extraktionslösung wird an $\mathrm{SiO}_{2}$ chromatographiert. Nach einer grünbraunen Zone, die ein nicht näher zu charakterisierendes Nebenprodukt in geringen Mengen liefert, läßt sich eine grüne Zone, die 3 in $38 \%$ Ausbeute enthält, eluieren. Die bei der Umkristallisation aus Toluol erhaltenen Kristalle enthalten pro Formeleinheit 1/2 Molekül Solvens. Elementaranalyse 3 $1 / 2$ Toluol: Gef.: C, 45.49; H, 3.51 . $\mathrm{C}_{22} \mathrm{H}_{20} \mathrm{Cr}_{4} \mathrm{~S}_{4}$ (620.62) ber.: $\mathrm{C}, 45.52 ; \mathrm{H}, 3.51 \%$. Molmasse 620.30 (FD-MS aus Toluol).

\section{Literatur}

1 J. Wachter, Angew. Chem., 101 (1989) 1645; Angew. Chem., Int. Ed. Engl., 28 (1989) 1613.

2 H. Brunner, J. Wachter, F. Guggolz, M.L. Ziegler und B. Balbach, J. Am. Chem. Soc., 104 (1982) 1765.

3 K. Endrich, E. Guggolz, O. Sherhadle, M.L. Ziegler und R.P. Korswagen, J. Organomet. Chem., 349 (1988) 323.

4 M.A. El-Hinnawi und A.K. Quaseer, J. Organomet. Chem., 296 (1985) 393; L.Y. Goh, T.W. Hambley und G.B. Robertson, Organometallics, 6 (1987) 1051.

5 W.A. Herrmann, J. Rohrmann und A. Schäfer, J. Organomet. Chem., 265 (1984) C1; W.A. Herrmann, J. Rohrmann, H. Nöth, Ch.K. Nanila und M. Draux, J. Organomet. Chem., 284 (1985) 189.

6 W. Chen, L.Y. Goh und T.C.W. Mak, Organometallics, 5 (1986) 1997.

7 Vorhergehende Veröffentlichung: H. Brunner, R. Graßl, J. Wachter, R. Nuber und M.L. Ziegler, J. Organomet. Chem., 431 (1992) 143.

8 J. Heck, K.A. Kriebisch und H. Mellinghoff, Chem. Ber., 121 (1988) 1753; W. Abriel und J. Heck, J. Organomet. Chem., 302 (1986) 363.

9 T.J. Greenhough, B.W.S. Kolthammer, P. Legzdins und J. Trotter, Inorg. Chem., 18 (1979) 3543; L.Y. Goh, T.W. Hambley und G.B. Robertson, J. Chem. Soc., Chem. Commun., (1983) 1458.

$10 \mathrm{In}\left(\mathrm{C}_{5} \mathrm{H}_{5}\right)_{2} \mathrm{Cr}_{2}(\mathrm{CO})_{4} \mathrm{~S}$ beträgt der Abstand der beiden $\mathrm{Cr}$-Atome voneinander ca. $4.00 \AA$, bei einem Winkel von 174.7(6) am Brückenschwefel [9].

11 H. Vahrenkamp, Angew. Chem., 87 (1975) 363; Angew. Chem., Int. Ed. Engl., 14 (1975) 322.

12 C. Wei, L.-Y. Goh, R.F. Bryan und E. Sinn, Acta Crystallogr., Sect. C, 42 (1986) 796.

13 A.A. Pasynskii, I.L. Eremenko, Y.V. Rakitin, V.M. Novotortsev, O.G. Ellert, V.T. Kalinnikov, V.G. Shklover, Y.V. Struchkov, S.V. Lindemann, T.K. Kurbanov und G.V. Gasanov, J. Organomet. Chem., 248 (1983) 309.

14 I.L. Eremenko, S.E. Nefedov, A.A. Pasynskii, B. Orazsakhatov, O.G. Ellert, Y.T. Struchkov, A.I. Yanovsky und D.V. Zagorevsky, J. Organomet. Chem., 368 (1889) 185. 
15 F. Bottomley, D.E. Paez, L. Sutin, P.S. White, F.H. Köhler, R.C. Thompson und N.P.C. Westwood, Organometallics, 9 (1990) 2443; F. Bottomley, J. Chen, S.M. MacIntosh und R.C. Thompson, Organometallics, 10 (1991) 906.

16 Wir danken Herrn Prof. K.G. Wieghardt (Bochum) für die Bestimmung der Temperaturabhängigkeit des magnetischen Moments. 